\title{
Multiple bilateral fibromas of the pleura
}

\author{
C. M. SCATTINI and A. ORSI \\ Hospital Rivadavia and Hospital Alvear, Buenos Aires, Argentina
}

\begin{abstract}
Scattini, C. M. and Orsi, A. (1973). Thorax, 28, 782-787. Multiple bilateral fibromas of the pleura. A patient with multiple bilateral fibromas of the pleura which evolved over 25 years is presented. Tumours were resected in four successive surgical interventions during this period. At the second operation 'spontaneous regression' of several small pleural fibromas noted at the first operation four years previously was observed. The differentiation between pleural fibroma and pleural localized mesothelioma is discussed.
\end{abstract}

Fibromas of the pleura are rare tumours. Even and Sors (1959) estimate that there is one primary pleural tumour for every 100 bronchial carcinomas and that in primary pleural tumours malignant lesions are four times more frequent than benign lesions. Reports of fibromas of the pleura only record the existence of single fibromas (Halperin, Bilesio, and Orlando, 1955; Decroix and Pieron, 1963; Sen, Parulkar, Kinare and Gandhi, 1964), but some authors report a recurrence (Kerr and Nohl, 1961) or a malignant transformation (Huguenin-Dumittan, Mach, and Press, 1968). We believe that our case of multiple bilateral benign fibromas of the pleura which evolved over a period of 25 years is unique.

\section{CASE REPORT}

O.P. de L., a 35-year-old woman, was first seen in January 1948 complaining of weakness and slight fever. Six months previously she had noticed pain in the posterior aspect of the right chest wall made worse by breathing. The pain was readily controlled with analgesics, but one month later bilateral chest pain developed as well as cough made worse by damp weather. In view of these symptoms a chest radiograph was taken which revealed an upper right paramediastinal mass (Figs. 1 and 2).

Physical examination showed evidence of weight loss with an evening fever of about $37 \cdot 5^{\circ} \mathrm{C}$. Physical examination was otherwise normal.

Laboratory investigations showed the blood count to be normal and the sedimentation rate $11 / 26$. Casoni reaction for hydatid disease was negative. Mantoux reaction $1 / 1,000$ was positive ++++ . She was treated with rest, calcium, and vitamins. Two months later the

Requests for reprints: Dr. C. M. Scattini, Hospital Rivadavia, Buenos Aires, Argentina patient stated that she had no more chest pain nor fever and had gained $5 \mathrm{~kg}$ in weight.

It was considered that the mediastinal mass observed radiographically could be due to a benign lung or mediastinal tumour. After induction of a right pneumothorax the tumour descended with the lung and this was taken as probable evidence that the mass arose from within the lung.

In April 1948 a right thoracotomy was performed and the tumour was felt in close relation to the right upper lobe, well delineated and surrounded by normal lung parenchyma. While exploring the rest of the lung and the parietal and visceral pleura, numerous

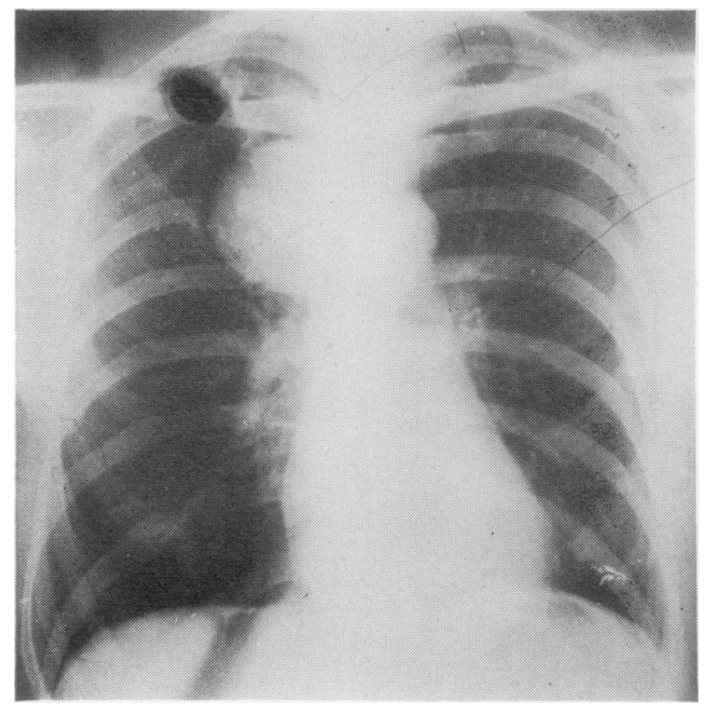

FIG. 1. Chest radiograph in 1948, showing right upper paramediastinal fibroma. 


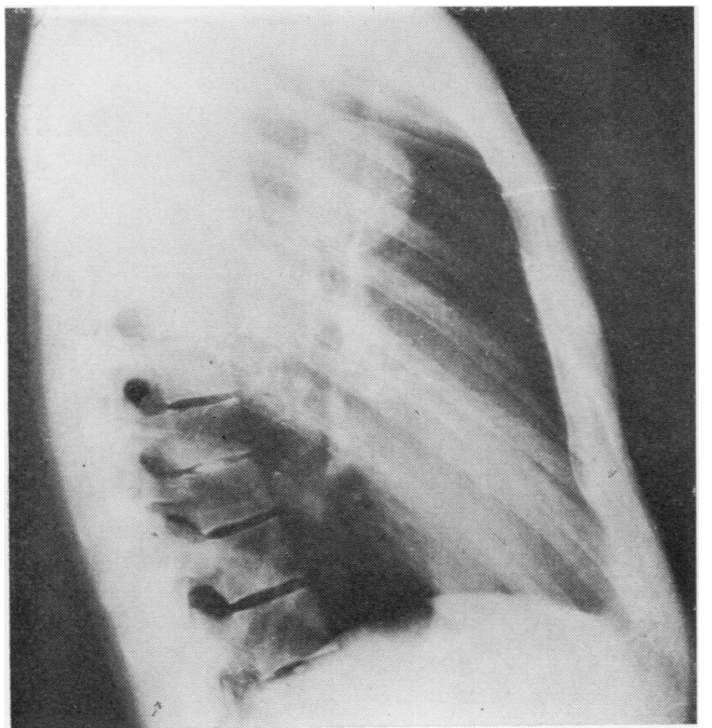

FIG. 2. Same patient as in Fig. 1. Lateral view. white hard nodules of different sizes were found over the visceral pleura and one over the parietal pleura. The largest nodule, approximately $4 \mathrm{~cm}$ long and $2 \mathrm{~cm}$ wide, was resected for histological study, and as all of these were considered to be metastases from either the primary pulmonary tumour or an undetected extrapulmonary source, resection of the main tumour was not undertaken. The histopathological diagnosis of the resected small tumour was benign fibroma of the pleura.

The postoperative course was normal but the patient's follow-up serial radiographs showed gradual growth of the main tumour. No enlargement of the small tumours observed during operation could be detected.

Four years later, in July 1952, in view of the progressive growth of the tumour, a second thoracotomy was carried out. A few pleural adhesions were easily divided. An unexpected finding was that from the multiple pleural seedlings observed at the previous operation, oniy one, the size of a grain of corn, whitish and hard, could be seen on the visceral pleura. On the surface of the visceral pleura small granulations, representing spontaneous involution of the tumours observed at the first operation, could be palpated but not seen. The main paramediastinal

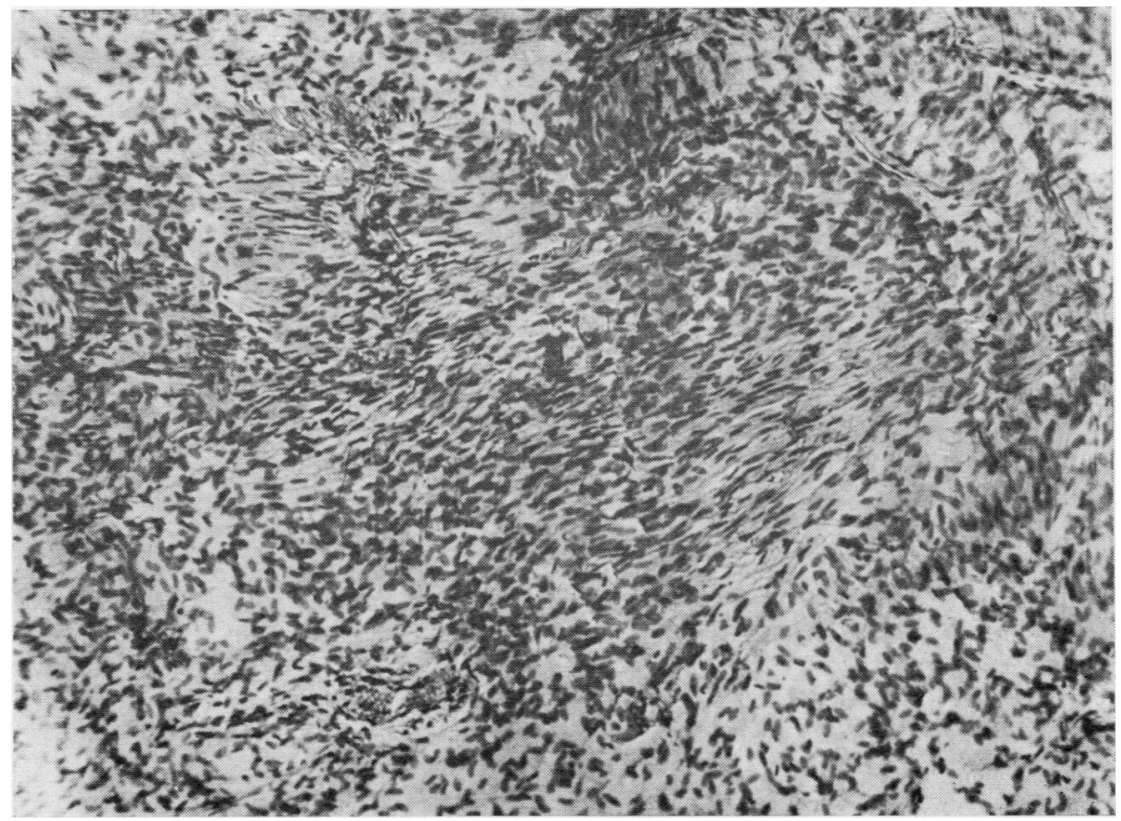

FIG. 3. Histology of fibroma resected in 1952. (Haematoxylin and eosin $\times 150)$. 
tumour, which arose from the visceral pleura and which had vascular pedicles arising from the lung parenchyma, was resected. Histopathological study showed, as in the case of the small tumour resected in the previous operation, that it was a benign fibrous pleural fibroma (Fig. 3).

Fifteen years after the second operation, in 1967, the patient, who up to that time had enjoyed good health, returned complaining of weight loss, weakness, and cough and a complete clinical evaluation was undertaken. A chest radiograph showed absence of the posterior end of the fourth and part of the fifth right ribs from the previous operations. In the lungs a number of new shadows were seen. The largest was in the right paratracheal region, and three others were in the left hemithorax. In addition, a marked elevation of the left hemidiaphragm was observed, which had not been noted in the previous radiographs. The left hemidiaphragm showed normal motility on screening. These radiographic shadows were interpreted as probably metastatic from an extrapulmonary tumour or as hydatid cysts. An exhaustive search for an extrapulmonary tumour was negative. The blood count, sedimentation rate, blood sugar and urea, serum protein electrophoresis, and urine were normal. Considering her previous operations 15 and 19 years previously, which on both occasions demonstrated that the tumours resected were pleural fibromas, it was considered probable that the new tumours were of a similar histological nature and it was decided to observe the patient.

Due to fortuitous circumstances, among them the

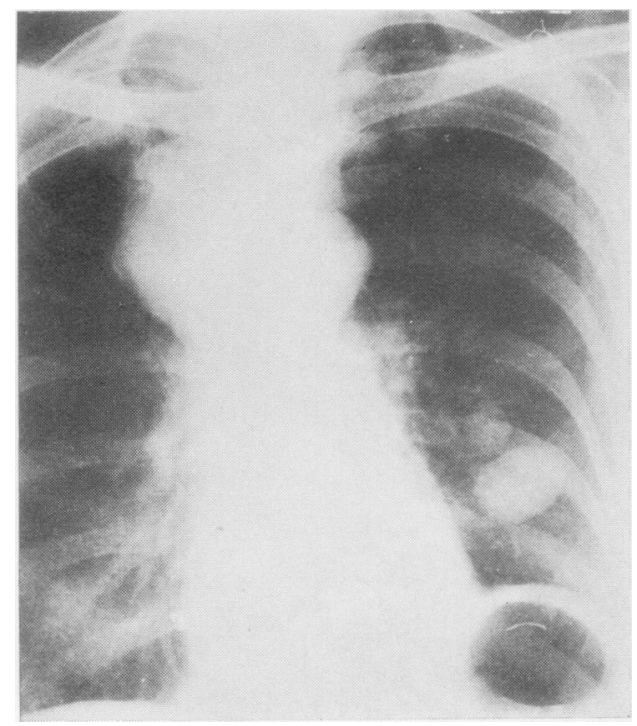

FIG. 4. Chest radiograph in 1970. A new tumour has grown in the right upper paramediastinal region, at the site of the fibroma resected in 1952. In the left hemithorax three other round tumours can be seen, one difficult to see in the $P A$ view.

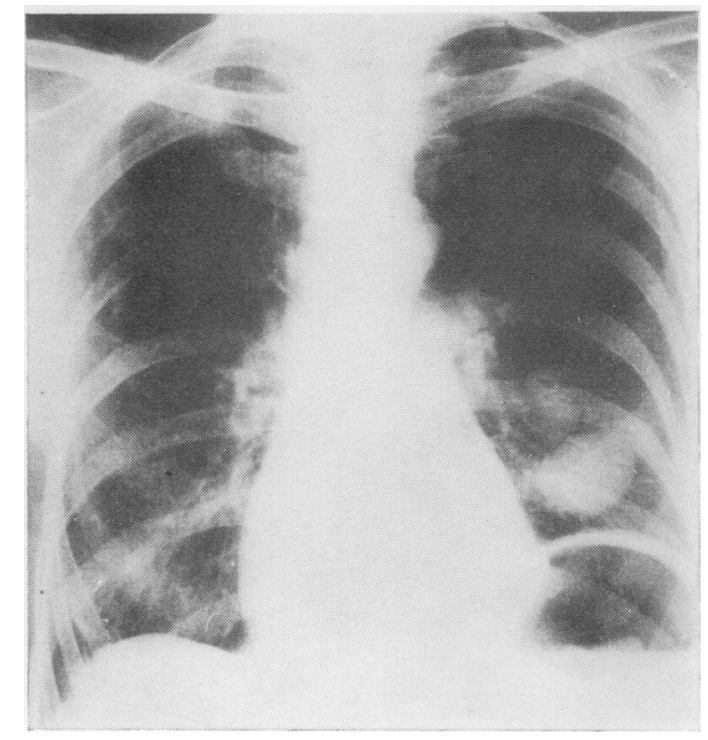

FIG. 5. Chest radiograph in 1972. Growth of the tumours in left hemithorax since 1970 is seen.

fact that the patient remained in perfect health, a further chest radiograph was delayed for three years. This chest radiograph (1970) showed a large right paratracheal mass, evidently the result of growth of the tumour observed three years previously. A barium swallow showed narrowing and displacement of the oesophagus by the tumour. There was also obvious growth of the three tumours in the left hemithorax (Fig. 4).

The growth noted during the previous three years and the compression of important mediastinal structures by the right paratracheal tumour indicated reoperation, after pulmonary function tests had been found to be normal. This operation (December 1970) was difficult as the right upper lobe was attached to the thoracic wall as a result of the previous operations, and, as the adhesions were vascular, haemostasis was difficult to achieve. After the lung had been freed, a large tumour growing into the lung but arising from the pleura could be seen, from which a small portion was resected for histological study by frozen section. This confirmed the assumption that it was a pleural fibroma. In the mediastinum the tumour abutted against the superior vena cava and the trachea, although it was not invading them. The tumour was resected; it measured $10 \times 8 \times 8 \mathrm{~cm}$.

Exploration of the surface of the right lung revealed two small flat tumours $4 \mathrm{~mm}$ in diameter on the visceral pleura of the middle and lower lobes. Both were resected and histological study showed them to be of a fibrous nature. Histopathological examination of the resected paratracheal tumour showed a pleural fibroma containing areas of stroma with extensive 


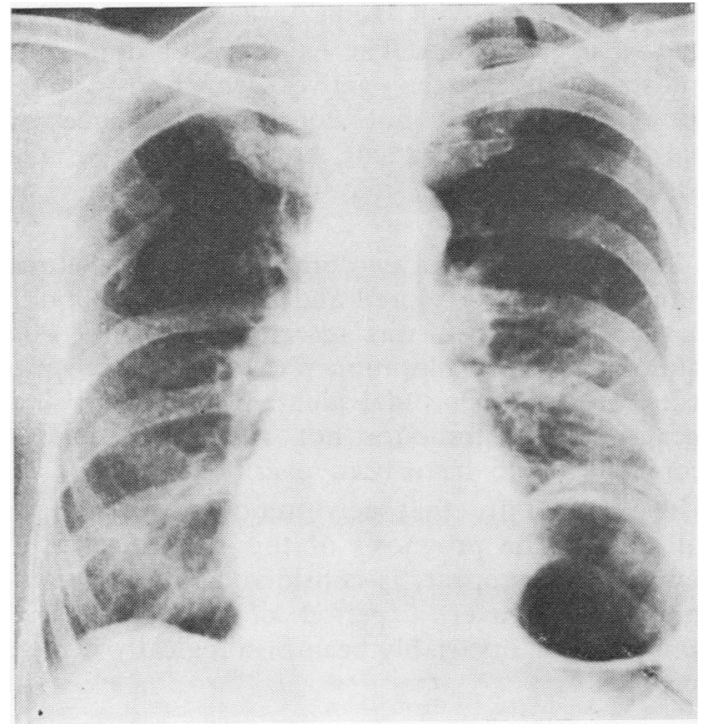

FIG. 6. Chest radiograph six months afier the last operation.

mucoid degenerations and a fibrous capsule in which frequent mitoses were observed.

A year and a half later obvious growth of the three tumours in the left hemithorax was seen (Fig. 5) and surgical resection was decided upon. The general health of the patient during this period was excellent and the laboratory studies and pulmonary function tests were completely normal. In July 1972 a left thoracotomy was performed. The lung was free of adhesions and on the upper lobe, in the area of the lingula, a well demarcated and superficial tumour was found, arising from the visceral pleura but penetrating into the lung. In order to remove it, it was necessary to resect part of the pulmonary parenchyma. A second tumour was found arising from the visceral pleura, well encapsulated, growing intraparenchymally, and measuring $2 \times 1 \times 1 \mathrm{~cm}$. In the posterior surface of the superior segment of the lower lobe, the third tumour that had been visible in the chest film was found, well encapsulated and measuring $3 \times 4 \times 4 \mathrm{~cm}$.

In addition to these three main lesions another three small round tumours of less than $0.5 \mathrm{~cm}$ in diameter were found on the visceral pleura, one on the upper lobe and the other two on the left lower lobe. They were locally resected. Histological examination showed the fibrous nature of all these tumours, some of them with areas of mucoid transformation and some with mitosis. Figure 6 shows a radiograph obtaired six months after the last surgical intervention.

\section{DISCUSSION}

This case presents two significant characteristicsthe spontaneous regression of most of the multiple pleural tumours observed at the first operation, and the multiplicity of fibromas over a period of almost 25 years. It is impossible to determine the cause of this tumour regression, although undeniable proof of spontaneous regression of malignant tumours does exist (Everson and Cole, 1959; Boyd, 1966). Although the determining mechanism is uncertain, there is no doubt that in this patient tumour regression occurred. The right paratracheal tumour discovered in the patient 18 years after her second operation had the same location as the fibroma resected then, and this raises the question whether it was a new tumour or a recurrence. The potential to recurrence of pleural tumours is well known, and in spite of the original benign fibrous nature of the tumour in this case the recurrence may present malignant characteristics. Kerr and Nohl (1961) described six patients with benign intrathoracic fibromas, judged by their postoperative histopathological appearances. In five, the tumour recurred with undeniably malignant characteristics; the remaining case showed a benign fibrous tumour. Recurrence took place up to 12 years after resection of the primary tumour.

Had there not been additional pleural tumours, the fibroma resected in 1970 might be regarded as a recurrence, but the possibility of a new tumour growing in almost the same place as the one previously resected could also be considered. It may be pointed out that the growth of fibromas seemed to be limited to the pleura, as an exhaustive examination of the patient did not reveal other fibromas elsewhere.

Numerous authors (Stout and Murray, 1942; Stout and Himadi, 1951; Benoit and Ackerman, 1953; Bilesio, Villamil, Gambarini, and Pasquini, 1958) consider fibrous tumours localized to the pleura as benign localized mesotheliomas, conceding that they derive from the mesothelium and that notwithstanding their benign fibrous appearance they are capable of malignant transformation, as shown by malignant change in some. It is possible to distinguish two types of pleural tibroma, depending on which of the histological structures of the pleura they originate from-the mesothelium or the pleural connective vascular tissue (Bracco, 1964). The former would retain its potential for malignant transformation, while the latter would remain benign, as any fibroma originating in the connective tissue elsewhere in the body. In our case, all the tumours resected during a 25-year period showed a benign fibromatous nature and the tolerance of the patient to the tumour seems to confirm its overall benign nature. 
Most pleural tumours are discovered at routine radiography (Galy, 1961). If on account of their size or location these tumours produce symptoms, the symptomatology is in general insidious. Pain is the most frequent symptom $(64 \%$ in the cases recorded by Biehl (1957)) and its location corresponds to where the tumour is located. Dyspnoea occurs in $50 \%$ and cough in $35 \%$ of cases. The dyspnoea is generally related to the size of the tumour and the cough is generally dry, of an irritating type, though haemoptysis may rarely occur. In some cases, especially when mediastinal compression occurs from large tumours, cyanosis, collateral circulation, and oedema may appear (Temple and Jones, 1954). In this event the barium swallow shows extrinsic compression of the oesophagus, though the patient may not complain of dysphagia. At surgery, compression of the upper vena cava by the tumour can be verified. Clagett, McDonald, and Schmidt (1952) found hypertrophic pulmonary osteoarthropathy in 16 of 24 cases of localized pleural mesotheliomas. They consider this to be a characteristic, and in some cases found a remarkable improvement of the osteoarthropathy following resection of the tumour.

The radiological appearances of pleural fibromas vary according to the location and size of the tumours. Generally they tend to be round, discrete, and of a uniform density. The differential diagnosis includes hydatid cysts, bronchial carcinoma, and loculated pleural collections. In our case, in the presence of multiple lung shadows, the most likely diagnosis was hydatid cysts (particularly in South America) or pulmonary metastasis from an extrapulmonary tumour. Nevertheless, the fact that the patient had been operated on many years before and a pleural fibroma found, and also that in a previous operation the existence of multiple pleural fibromas had been verified, suggested that the new radiological shadows were pleural fibromas. Moreover, the radiographic and tomographic studies performed showed that all the tumours were in close contact with the pleural surface and in no case isolated in the depth of the pulmonary parenchyma.

In our case, each of the tumours surgically resected had developed from the visceral pleura although some had grown into the lung. Tumours arising from the visceral pleura are more likely to be benign than those arising from the parietal pleura (Friedman, 1950).

Each tumour was well encapsulated, which permitted easy enucleation. Histologically they showed a monoformic proliferation of connective tissue elements surrounded by fascicles of collagen- reticular fibres with some areas of extensive mucoid degeneration. The existence of numerous mitoses explains the active growth of these tumours and does not constitute evidence of malignancy in this patient, but in other cases the existence of mitoses may constitute a sign of malignancy.

In the patient's last two operations both pleuras were thoroughly explored and every small tumour that could be seen was resected. However, one cannot say that exploration was complete because access to the mediastinal pleura was difficult and because small tumours not detectable macroscopically could have been overlooked.

The possibility that new tumours will appear still exists. The prognosis of this patient, even if new tumours appear, is considered to be favourable because over a period of 25 years these tumours have invariably been histologically benign fibromas.

\section{REFERENCES}

Benoit, H. W. and Ackerman, L. V. (1953). Solitary pleural mesotheliomas. Journal of Thoracic Surgery, 25, 346.

Biehl, F. (1957). Contribution à l'étude des tumeurs primitives de la plèvre. Thesis, University of Nancy.

Bilesio, E. A. Villamil, E. F. J., Gambarini, A., and Pasquini, A. T. (1958). Fibromesotelioma solitario de pleura parietal. La Prensa Médica Argentina, 45, 1343.

Boyd, W. (1966). The Spontaneous Regression of Cancer. Thomas, Springfield, Illinois.

Bracco, A. N. (1964). Mesoteliomas malignos de la pleura. Revista de la Asociación Médica Argentina, 78, 74.

Clagett, O. T., McDonald, J. R., and Schmidt, H. W. (1952). Localized fibrous mesothelioma of the pleura. Journal of Thoracic Surgery, 24, 213.

Decroix, G. and Pieron, R. (1963). Contribution a l'étude des fibromes de la plèvre. Bulletins et Memoires de la Société Médicale des Hôpitaux de Paris, 114, 97.

Even, R. and Sors, C. (1959). Les mésothéliomes. Essai de classification anatomo-clinique des tumeurs pleurales primitives. Presse Médicale, 67, 2058.

Everson, T. C. and Cole, W. H. (1959). Spontaneous regression of malignant disease. Journal of the American Medical Association, 169, 1758.

Friedman L. L. (1950). Tumors of the pleura. Diseases of the Chest, 17, 756.

Galy, P. (1961). Le diagnostic des tumeurs pleurales primitives ou soit-disant telles. Journal de Médecine de Bordeaux, 138, 1261.

Halperin, A., Bilesio, E. A., and Orlando, E. A. (1955). Fibromesotelioma solitario de pleura visceral. Prensa Médica Argentina, 42, 1031.

Huguenin-Dumittan, S. A., Mach, R. S., and Press, P. (1968). Fibro-mésothéliome 'bénin'. Schweizerische medizinische Wochenschrift, 98, 1368.

Kerr. W. F. and Nohl, H. C. (1961). Recurrence of 'benign' intrathoracic fibromas. Thor ax, 16, 180. 
Sen, P. K., Parulkar, G. B., Kinare, S. G., and Gandhi, R. K. (1964). Giant pleural fibroma. British Journal of Surgery, 51, 636.

Stout, A. P., and Himadi, G. M. (1951). Solitary (localized) mesothelioma of pleura. Annals of Surgery, 133, 50.
_- and Murray, M. R. (1942). Localized pleural mesothelioma. Archives of Pathology, 34, 951.

Temple, L. J. and Jones, G. P. (1954). Two cases of giant intrathoracic fibroma. Thorax, 9, 112. 